\title{
Design of USB3.0 Video Transmission System with UVC Protocol Supported
}

\author{
JiangBo $\mathrm{Li}^{1, \mathrm{a}}$, ChunTing Ma ${ }^{1, \mathrm{~b}}$, Fan Yang $^{1, \mathrm{c}}$ \\ ${ }^{1}$ Ordnance Engineering College, Shijiazhuang 050000, China; \\ a15710323796@163.com, bempty_set@126.com, '1686046416@126.com
}

Keywords: USB3.0, UVC, video transmission.

\begin{abstract}
A USB3.0 video transmission system with UVC protocol supported is designed. CMOS image sensor is used as photoelectric device. As a USB3.0 controller chip, CYUSB3014 acquires and processes data from CMOS image sensor, then converts the data to video that conforms to the UVC protocol. At last the video data display on computer real-time. The system implements video transmission with UVC protocol supported in high speed and resolution in industrial video.The design with clear structure could be not only used as a handheld device, but also a debug equipment of several types of image sensor.
\end{abstract}

\section{Introduction}

With the development of science and technology, image sensor has a great improvement in performance, while the use of image sensor is still inconvenient for the average user. For example, the user must install drivers[1].etc. When the image sensors with high speed and high-resolution begin to appear, it is difficult to overcome the difficulties in the short time.

In this paper, the CMOS image sensor OV5640AF of OV Company is used for image data acquisition, and the chip CYUSB3014, which is USB3.0 controller of Cypress, is as the transmission channel. The image data are converted into the video data with UVC protocol supported and uploaded to PC to display real-time. This design is compact and can be used to test various types of image sensors. The Video Transmission System can accomplish image data acquisition and display real-time. The advantage is that the image data collected has no use for any compression encoding, and it can be connected to the computer without the installation of drivers. The computer control interface can be easily set up for various types of arguments of image sensors, which is easier for users[2]. Its application focuses on monitoring the small filed and internal space which is difficult to observe by human eyes, it also can analyze the image data according to the actual desire.

\section{Overall System Design}

The system is composed of three elements: image acquisition, transmission control and image display unit( Fig. 1).

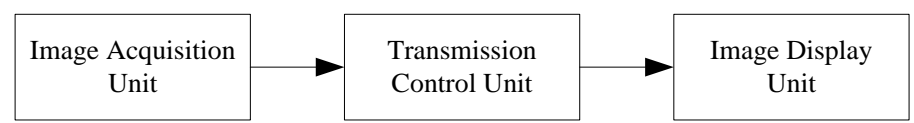

Fig.1 System structure diagram

The image acquisition unit is a CMOS image sensor, which acquire the image data and transmit to the next unit. The unit[3] use OV5640AF of OV company, which is a 5,000,000 pixel CMOS image sensor, the maximum resolution is $2592 \times 1944$, and it support for a variety of video output format; The sensor can be initialized and configured by the $\mathrm{I}^{2} \mathrm{C}$ bus.

The CYUSB3014 is responsible for the transmission control, which finish two jobs: first, initialize the CMOS image sensor, second, receive and process the image data and transmit to the computer.

The image display unit is the computer which receives image data and display on the screen.

The system hardware consists of three parts (Fig. 2): image sensor USB, CYUSB3014,OV5640AF and the computer. 


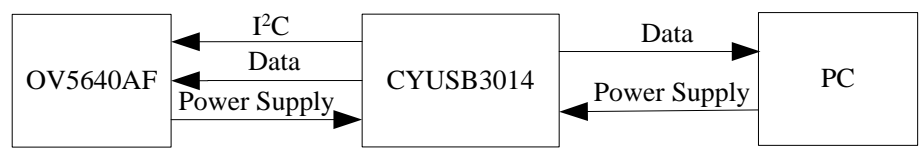

Fig.2 Hardware connection diagram

\section{Video Acquisition Unit}

Initialization of Image Sensor. In order to make Image data from image sensor OV5640AF to dispaly, there is two aspects of work to be completed:

For the first, the power supply of image sensor is required: $2.8 \mathrm{~V}$ and $1.8 \mathrm{~V}$, produced from the main power supply (the system power supply for USB, 5V) with the chip CAT6219.

Second, the initialization of image sensor need to be achieved, the chip CYUSB3014 operate the internal registers of image sensor and complete the work through the $\mathrm{I}^{2} \mathrm{C}$ interface.

Note: Although the CYUSB3014 chip has been integrated with the $\mathrm{I}^{2} \mathrm{C}$ interface, it has been proved that the $\mathrm{I}^{2} \mathrm{C}$ interface can not automatically resume high level when the slave device could not respond, so it is be good choice to driver any two idle IO pins to simulate the $\mathrm{I}^{2} \mathrm{C}$ interface.

The initialization of the image sensor OV5640AF consists of the power supply part and the initialization part (Fig. 3).

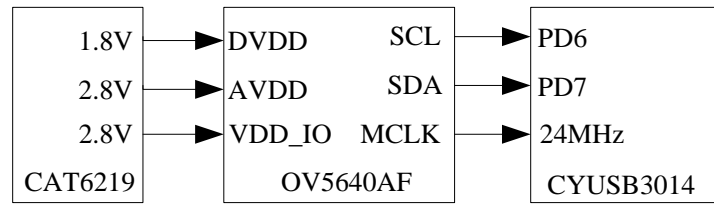

Fig.3 Initialization connection diagram of OV5640AF

Image Sensor Initialization Steps. the $\mathrm{I}^{2} \mathrm{C}$ interface SCL (serial clock), SDA (data line) read or write registers of OV5640AF, get or change the sensor's various parameters (gain, speed, size, etc.).The initialization of the image sensor (Figure 4) comprise 5 steps:

(1) power supply. Three kinds of power: digital power supply (DVDD), analog power supply (AVDD) and IO power supply (DOVDD), there is no strict sequence between the three kinds of power.

(2) the RESET, MCLK and PWDN pins of the image sensor are examined in turn.

(3) the version number of the image sensor is read, and if it is in line with the version of the product, it is capable of working on behalf of the $\mathrm{I}^{2} \mathrm{C}$ bus.

(4) assignment of registers and variables complete a series of work, such as the configuration of PLL, and the resolution and format of the output data.

Notice: when some changes appear in writing the registers of the image sensor, it is necessary to pay attention to the delay, if necessary, the register can be writed for many times.

(5)check the OV5640AF pin: VSYNC (frame signal), HSYNC (Line signal), Data[0:7] (8 bit image data) whether there is a waveform output, if yes it is proved that the image sensor OV5640AF has been successfully initialized.

\section{Transmission Control Unit}

The main task of this unit is to complete the initialization of USB chip CYUSB3014, and bring the command to computer, receive the image data from the image sensor. The image data is parsed into data packets with the UVC protocol supported.

Firmware Design of USB Chip. Image data is received from the image sensor and brought to computer with a UVC protocol supported. Receive the request command and send the command to the image sensor to respond the request. Including the construction of two DMA transmission channels, change to another channel to continue to store data when the current transmission channel 
cache memory is full of data; Design GPIF II transmission state machine and process upper machine's request, including video brightness, contrast control, etc..

Video Data Transmission with UVC supported. Through the USB control port, host communicates with devices that conform of the UVC protocol and transmit corresponding control and video stream request. The peripheral devices respond to these requests to query and change the related control properties. The UVC protocol defines these video related controls as a function that you can use to change the properties of the image or video stream. A function can be a video control properties, such as brightness, contrast, color or a video flow properties, such as color format, frame size, frame rate. Function can be configured to inform the host computer by the UVC USB configuration descriptor. Each function has its own properties.

USB Firmware Analysis. The overall framework of the firmware is shown (Fig. 4):

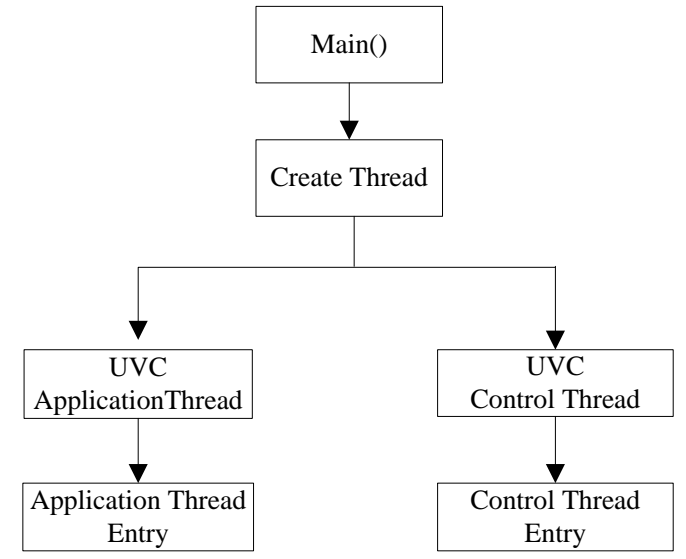

Fig. 4 Framework of the firmware

In the main program, main task is the clock and initialization with RTOS ThreadX. Then create two threads for the use of the firmware: one is the UVC application thread, mainly used to receive image data; the other is the UVC control thread, mainly used to respond the request of the user. The UART port is initialized and the DMA channel is constructed, and the Endpoint port is configured in the application thread entry function. The image's luminance and contrast is stetted in the UVC control thread entry function. The flow chart (Figure 5) is as follows:

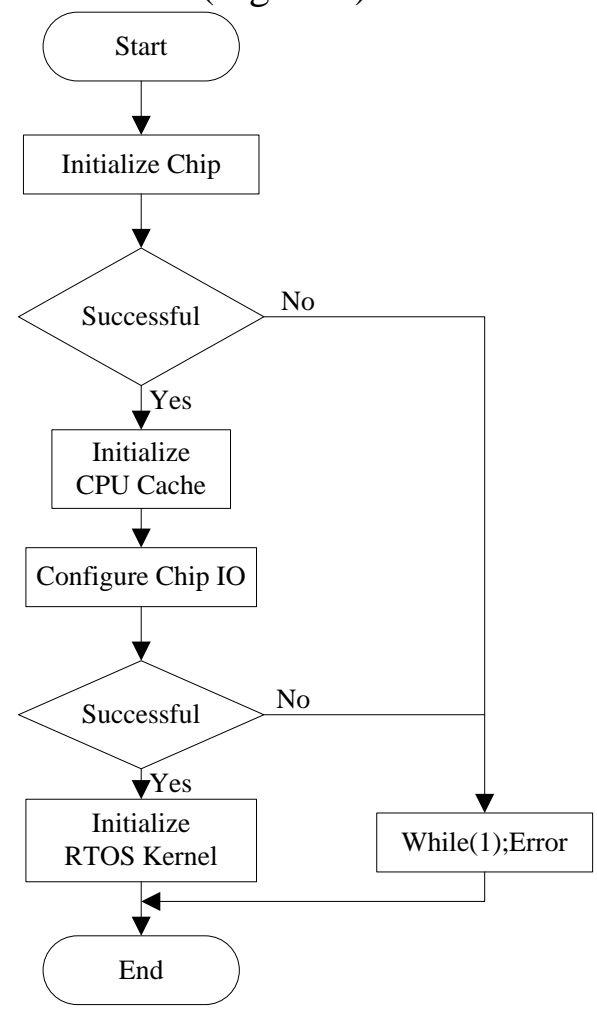

Fig. 5 Flow chart of main program 


\section{Summary}

Aiming at improving the application of image sensor, the video transmission system is designed,which is based on USB3.0 transmission control. The video data from the image sensor is converted to the data with the UVC protocol.

To ensure high speed[6] of video transmission, the two DMA channels are constructed in the firmware using the ping pong transmission mode. In addition, the transmission state machine is designed. The hardcore determine the general programmable interface (GPIF II) signal, receive and store video data transmitted from the image sensor, complete the conversion of two DMA channels, generate the end signal of the frame.

The result of experiment has shown that the system has good availability with the UVC protocol supported. The image which display on the computer is clear and stable with the frame rate up to 10fps,and the transmission speed can reach up to a maximum of $768 \mathrm{Mbps}$.

\section{References}

[1] HAN Juntao,GUAN Yong, Implementation of SoC-PC Communication Interface Based on USB2.0.IEEE International Conference on New Trends in Information and Service Science. (2009) 831-834.

[2] G. Raja Raman, M. Sarath Chandran, and S. R. vinotha. Motion Based Security Alarming System for Video Surveillance.Planetary Scientific Research Center Conference Proceedings Volume 1:6-10.

[3] OV5640AF_datasheet on http://www.ovt.com

[4] AN75779-How to Implement an Image Sensor Interface with EZ-USB $\mathbb{Q}$ FX3 in a USB Video Class (UVC) Framework. http://www.cypress.com

[5] Xu Dacheng, Zhao Xiaofei, Design and Implementation of The Image Quality Analyzing System of CMOS Camera. IEEE The Eighth International Conference on Electronic Measurement and Instruments,2007,pp.97-100.

[6] Gibbons, Karp. B, Ke. Y, Nath. S. IrisNet:an architecture for a worldwide sensor Web.USA:Pervasive Computing. (2003) 